\title{
Interspecific Hybridization between Aspergillus nidulans and Aspergillus rugulosus by Fusion of Somatic Protoplasts
}

\author{
By F. KEVEI* AND J. F. PEBERDY \\ Department of Botany, University of Nottingham, Nottingham $N G_{7} 2 R D$
}

(Received 25 February 1977; revised 13 May 1977)

\begin{abstract}
Interspecific heterokaryons have been produced between auxotrophic mutants of Aspergillus nidulans and Aspergillus rugulosus by polyethylene glycol induced fusion of somatic protoplasts. The heterokaryons grew very slowly producing colonies of irregular shape and, on complete medium, the parental strains were readily segregated. However, during longterm cultivation on minimal medium many of the heterokaryons gave rise to vigorously growing sectors characterized by the secretion of a brown pigment and by their stability when subcultured on complete medium. Protoplasts isolated from the heterokaryons gave rise to the same new colony type when regenerated on minimal medium, and to the new colony type plus the parental types on complete medium. The new colony type was assumed to be an interspecific 'hybrid' and showed normal vegetative morphology, regular colony shape and size but produced few conidia. Conidia formed early in the development of the 'hybrid' gave rise to 'hybrid' colonies. Various sizes of conidia were observed; the largest were uninucleate and the smallest were enucleate. Comparisons of conidial size, numbers of nuclei in cells and DNA content per nucleus for 'hybrid' and parental strains indicated that the 'hybrid' was diploid. Conidia from older cultures of the 'hybrid' were heterogeneous with 'hybrid', parental and recombinant colony types developing on germination. Cultivation of the 'hybrid' in the presence of benomyl gave segregation of parental and recombinant sectors.
\end{abstract}

\section{INTRODUCTION}

In the Fungi interspecific hybrids have been obtained in Sordaria (Lewis, 1969), Neurospora (Dodge, 1936) and in Verticillium (Hastie, 1973) by conventional sexual and parasexual events. Developments in the field of somatic cell genetics utilizing cell fusion in animal systems (Ruddle \& Creagan, 1975) and protoplast fusion in higher plants (Carlson, 1973; Cocking, 1972) have provided the impetus for similar studies in fungi. These simple eukaryotes exhibit many characteristics which make them good systems for this area of investigation, in particular the availability of auxotrophic mutants makes selection of heterokaryons, hybrids and recombinants relatively easy. Techniques for protoplast isolation and culture are well established and more recently methods for fusion have been published (Anné \& Peberdy, 1975, 1976; Ferenczy, Kevei \& Szegedi, 1975a). A possible barrier to hybridization in fungi is the hyphal wall and its removal at protoplast formation may permit more extensive interaction between species than known hitherto. More recent reports suggest this is the case; Anné, Eyssen \& De Somer (1976) isolated interspecific hybrids in crosses between Penicillium chrysogenum and $P$. roquefortii and Ferenczy, Szegedi \& Kevei (1977) have achieved similar results with Aspergillus nidulans and A. fumigatus. In both these instances the segregation properties of the hybrids suggested they were aneuploid.

* Present address: Department of Microbiology, Attila Jozsef University, Szeged, P.O. Box 428, Hungary. 
This paper describes results in which more closely related species were used for hybridization, namely $A$. nidulans and $A$. rugulosus, which both belong to the $A$. nidulans species group (Raper \& Fennell, 1965).

\section{METHODS}

Organisms and growth conditions. The strains of $A$. nidulans and $A$. rugulosus used in these experiments are listed in Table I. They were maintained on the complete medium (CM) of Ferenczy, Kevei \& Szegedi (1975 b). The two species show several distinct morphological features. The colony of $A$. nidulans is fast growing and has a spreading habit, while $A$. rugulosus grows more slowly and is buckled and wrinkled. Aspergillus nidulans produces abundant conidia, but in $A$. rugulosus conidiophores are sparse and spores are few. Cleistothecia are very abundant in $A$. rugulosus forming several layers in the mycelium; they are also abundant in $A$. nidulans but more generally scattered over the colony.

Trichoderma harzanium CBs345-33 was obtained from Professor J. G. H. Wessels, University of Groningen, The Netherlands, and was maintained on a glucose; salts medium (Wessels, 1965). All cultures were incubated at $30^{\circ} \mathrm{C}$.

Protoplast formation. Protoplasts were prepared from all strains using the procedure described by Ferenczy et al. (1975a). The lytic enzyme used was prepared from $T$. harzanium following the method of Peberdy \& Isaac (1976). In some experiments the Trichoderma enzyme was supplemented with freeze-dried Helix pomatia digestive juice ( $1 \%, w / v)$ extracted from snails collected in Szeged, Hungary.

Protoplast fusion. After washing in osmotic stabilizer (Ferenczy et al., 1975a), protoplasts from the two different strains to be fused were mixed $\left(1 \times 10^{6}\right.$ protoplasts $\mathrm{ml}^{-1}$ for each strain). Fusion was induced using polyethylene glycol (PEG) of mol. wt 4000 (Koch-Light) following the procedure described by Ferenczy et al. (1975b). Protoplasts from fusion mixtures were plated at suitable dilutions on to Pontecorvo minimal medium (MM; Pontecorvo et al., 1953) supplemented with $0.6 \mathrm{M}-\mathrm{KCl}$ and on to $\mathrm{CM}$, similarly supplemented. Colonies derived from fusion products were maintained on $\mathrm{MM}$. The fusion frequency was calculated from the ratio of colonies growing on MM and CM.

Nuclear staining. For nuclear staining, protoplasts were fixed in ethanol/acetic acid $(\mathrm{I}: \mathrm{I}, \mathrm{v} / \mathrm{v})$, washed and then stained in acridine orange ( $0.1 \%, w / v)$ using the technique of Clutterbuck \& Roper (1966). Spores were fixed and stained in a similar manner.

DNA estimation. The DNA content of protoplasts was estimated using the method of Bostock (1970). Samples $(\mathrm{I} \mathrm{ml})$ of suspensions containing $1 \times 10^{\circ}$ protoplasts $\mathrm{ml}^{-1}$ from parental strains or 'hybrids' were used. The nuclear content was determined by counting using protoplasts stained as described above.

Marker characterization of segregants. The presence of auxotrophic markers in segregants was determined using MM supplemented with the specific requirements. Spore colour was also noted but the $t s$ marker was not scored and will not be referred to in subsequent genotypes.

\section{RESULTS AND DISCUSSION}

\section{Isolation of interspecific heterokaryons}

Colonies developing from fusion mixtures plated on MM and CM are shown in Fig. I. Interspecific heterokaryons were isolated from all fusion mixtures at frequencies up to $50 \%$ of those obtained in an $A$. nidulans intraspecific fusion (Table 2). The fusion frequencies obtained in these experiments were higher than reported earlier for intraspecific fusion in various aspergilli and penicillia (Anné \& Peberdy, 1976). A probable cause of this difference is the size of the colony-forming units following PEG treatment. Anné \& Peberdy (1976) used a washing procedure after exposure to $P E G$ and it is likely that this would break up the protoplast aggregates thereby reducing the likelihood of fusion events, as shown by colony development on $\mathrm{MM}$, but increasing the number of colonies developing on $\mathrm{CM}$.

Heterokaryons derived from various fusion mixtures showed irregular colony shape and their size and morphology differed from the parental colony forms on CM or MM supplemented with the required nutritional factors. Heterokaryon formation on $\mathrm{MM}$ also occurred where the two parental strains had similar nutritional requirements (i.e. NI + RI and N2 +R2) indicating that the independent mutations complemented each other.

Several control experiments were set up with platings on both MM and CM: (i) protoplasts of the two parental strains treated with PEG and plated separately; (ii) mixtures of protoplasts of the two parental strains without PEG treatment; (iii) mixtures of lysed 
Table I. Strains used

\begin{tabular}{|c|c|c|c|}
\hline Species & Strain no. & Marker genotype* & Origin \\
\hline $\begin{array}{l}\text { Aspergillus } \\
\text { nidulans }\end{array}$ & $\begin{array}{l}\mathrm{NI} \\
\mathrm{N} 2 \\
\mathrm{~N} 3\end{array}$ & $\begin{array}{l}\text { pabar, } y ; \text { proz; } t s 6 \\
\text { pabar, } y ; \text { met2;ts6 } \\
\text { pabaI, y;lyst;ts6 }\end{array}$ & $\left\{\begin{array}{l}\text { Mutants induced (u.v.) in } A . \text { nidulans R2 I } \\
\text { (pabar, } y: \text { ts6), obtained from } \\
\text { Dr R. F. Rosenberger }\end{array}\right.$ \\
\hline $\begin{array}{c}\text { Aspergillus } \\
\text { rugulosus }\end{array}$ & $\begin{array}{l}\mathbf{R I} \\
\mathbf{R} 2\end{array}$ & $\begin{array}{l}\text { proI } \\
\text { metI }\end{array}$ & ants induced (u.v.) in $A . r$ \\
\hline
\end{tabular}

- lys, Requirement for lysine; met, requirement for methionine; paba, requirement for $p$-aminobenzoic acid; pro, requirement for proline; $y$, yellow conidia; $t s$, temperature-sensitive growth.
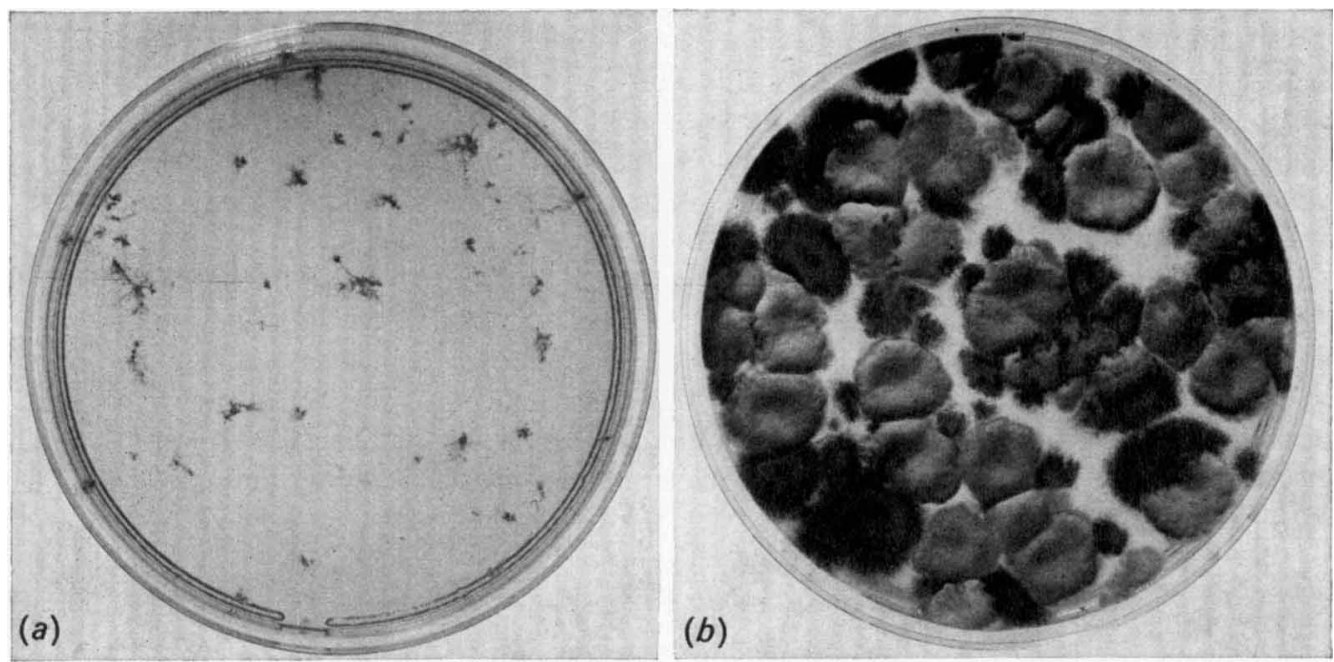

Fig. I. Five-day-old cultures developed from a fusion mixture of $A$. nidulans strain NI (pabaI, $y$; proz) and $A$. rugulosus strain R2 (metr) plated on (a) stabilized MM (1 $\times 10^{-3}$ dilution); and $(b)$ stabilized $\mathrm{CM}\left(\mathrm{I} \times \mathrm{IO}^{-4}\right.$ dilution). The plating on $\mathrm{MM}(a)$ has produced slow-growing interspecific heterokaryons while only the parental types are developing on CM $(b)$.

\section{Table 2. Frequencies of heterokaryon formation after polyethylene glycol treatment of protoplast mixtures}

Protoplasts from various parent strains were mixed in $30 \%(\mathrm{w} / \mathrm{v})$ PEG in $0.1 \mathrm{M}-\mathrm{CaCl}_{\mathrm{z}}$ at room temperature for $10 \mathrm{~min}$. Dilutions were plated on to osmotically stabilized $\mathrm{MM}$ and $\mathrm{CM}$.

$$
\text { Fusion mixture Fusion frequency }(\%)^{*}
$$

\begin{tabular}{|c|c|c|}
\hline \multirow[t]{6}{*}{ Interspecific } & $N I+R I$ & $5 \cdot 58 \pm I \cdot 74$ \\
\hline & $\mathrm{NI}+\mathrm{R} 2$ & $6 \cdot 99 \pm 1 \cdot 76$ \\
\hline & $\mathrm{N} 2+\mathrm{RI}$ & $2 \cdot 23 \pm I \cdot 08$ \\
\hline & $\mathrm{N} 2+\mathrm{R} 2$ & $2.76 \pm 0.93$ \\
\hline & $\mathrm{N}_{3}+\mathrm{RI}$ & $4 \cdot 02 \pm I \cdot 23$ \\
\hline & $\mathrm{N} 3+\mathrm{R} 2$ & $4 \cdot 52 \pm 1 \cdot 47$ \\
\hline Intraspecific & $\mathrm{NI}+\mathrm{N} 3 \dagger$ & $13.71 \pm 3.28$ \\
\hline
\end{tabular}

* Ratio of numbers of colonies growing on $\mathrm{MM}$ and $\mathrm{CM}$.

$\dagger \mathrm{MM}$ was supplemented with $2 \mu \mathrm{g} p$-aminobenzoic acid $\mathrm{ml}^{-1}$. 
protoplasts; and (iv) mixtures of ungerminated conidia with or without PEG treatment. In no case were heterokaryons obtained. Attempts to produce heterokaryons by other conventional procedures (Pontecorvo et al., 1953; Barron \& MacNeil, 1962) were also unsuccessful.

\section{Behaviour of the interspecific heterokaryons and 'hybrid' development}

When cultured on $\mathrm{CM}$ the heterokaryons segregated the parental strains very readily (Fig. 2a). On MM (Fig. $2 b$ ) the colonies developed slowly having an irregular shape and producing only few conidia which on germination produced colonies of the parental strains. Segregation was detected on the basis of both the striking differences in parental colony and the nutritional markers. After 10 to 14 days' incubation some of the heterokaryons on MM started to grow more vigorously producing colonies of normal shape and size and more stable than the heterokaryons (Fig. 3). The various heterokaryons obtained showed differences in their ability to yield these 'hybrids'. Those of cross NI +R2 were the most vigorous in respect of 'hybrid' formation, N3+R2 less so and N3+RI heterokaryons failed to yield 'hybrids'. A clear correlation existed between fusion frequency and incidence of 'hybrid' formation. Development of these 'hybrids' is presumably a consequence of interaction between parental nuclei in the heterokaryotic hyphae.

Protoplasts prepared from heterokaryons cultured on MM gave rise to the new type of 'hybrid' colonies when regenerated on osmotically stabilized MM and to both 'hybrid' and parental colonies on stabilized CM (Table 3). The differences in frequencies of the various colony types cannot be explained at this stage, but may reflect the ratio of parental nuclei in the fusion products and the parental biochemical deficiencies. In the NI $+R 2$ heterokaryon, the number of 'hybrid' colonies obtained was affected by the age of the culture used for protoplast isolation.

\section{Behaviour and stability of 'hybrids'}

Hyphae of the 'hybrids' were identical to the parental form, but showed a pattern of branching similar to $A$. nidulans. The mycelium of the 'hybrid' colonies was grey-white and the conidia were white. On CM or MM the 'hybrid' colonies produced a brown pigment which was released into the medium. No pigmentation occurred and the colonies were white, when grown on minimal medium with $\left(\mathrm{NH}_{4}\right)_{2} \mathrm{SO}_{4}$ in place of $\mathrm{NaNO}_{3}$ (Ferenczy et $a l ., 1975 b)$, suggesting that pigmentation was influenced by nitrogen metabolism. Neither the parental strains nor the interspecific heterokaryons produced pigment.

During the early stages of culture on CM the 'hybrids' appeared to be stable with no evidence of segregation by sectoring. Protoplasts isolated from 'hybrids' grown on CM for up to 5 days regenerated only 'hybrid' colonies on CM. The 'hybrids' produced few conidia (about $2 \times 10^{3}$ to $2 \times 10^{4}$ conidia $\mathrm{cm}^{-2}$ ) and the conidial apparatus showed a reduced structure. Conidia formed during the first 5 days of culture on CM or MM were of two sizes. The larger had an average diameter of $5 \cdot 78 \pm \mathrm{I} \cdot 54 \mu \mathrm{m}$; the smaller were similar in size to those of the parents $(3.75 \pm 0.78 \mu \mathrm{m}$ for NI and $3.83 \pm 0.19$ for R2). The larger spores were uninucleate and germinated to give 'hybrid' colonies while the smaller spores were enucleate.

As the colonies aged, particularly those grown on CM, individual or small clusters of conidiophores developed which were sites of spontaneous segregation. These conidiophores showed the normal morphology of either $A$. nidulans or $A$. rugulosus and were further distinguished by bearing yellow or green spores respectively. The conidia were similar in diameter to those of the parents and they were uninucleate. Conidia prepared from I0-dayold cultures of 'hybrids' from NI +R2, N3 + RI and N3 + R2 grown on CM or MM gave 'hybrid', parental and a variety of other segregant colonies when plated on CM or supplemented MM (Table 4).

The N3 + RI 'hybrid' grown on MM gave predominantly 'hybrid' subcultures, while cultures on CM produced 'hybrid' and parental subcultures in about a I : I ratio (Table 4). 

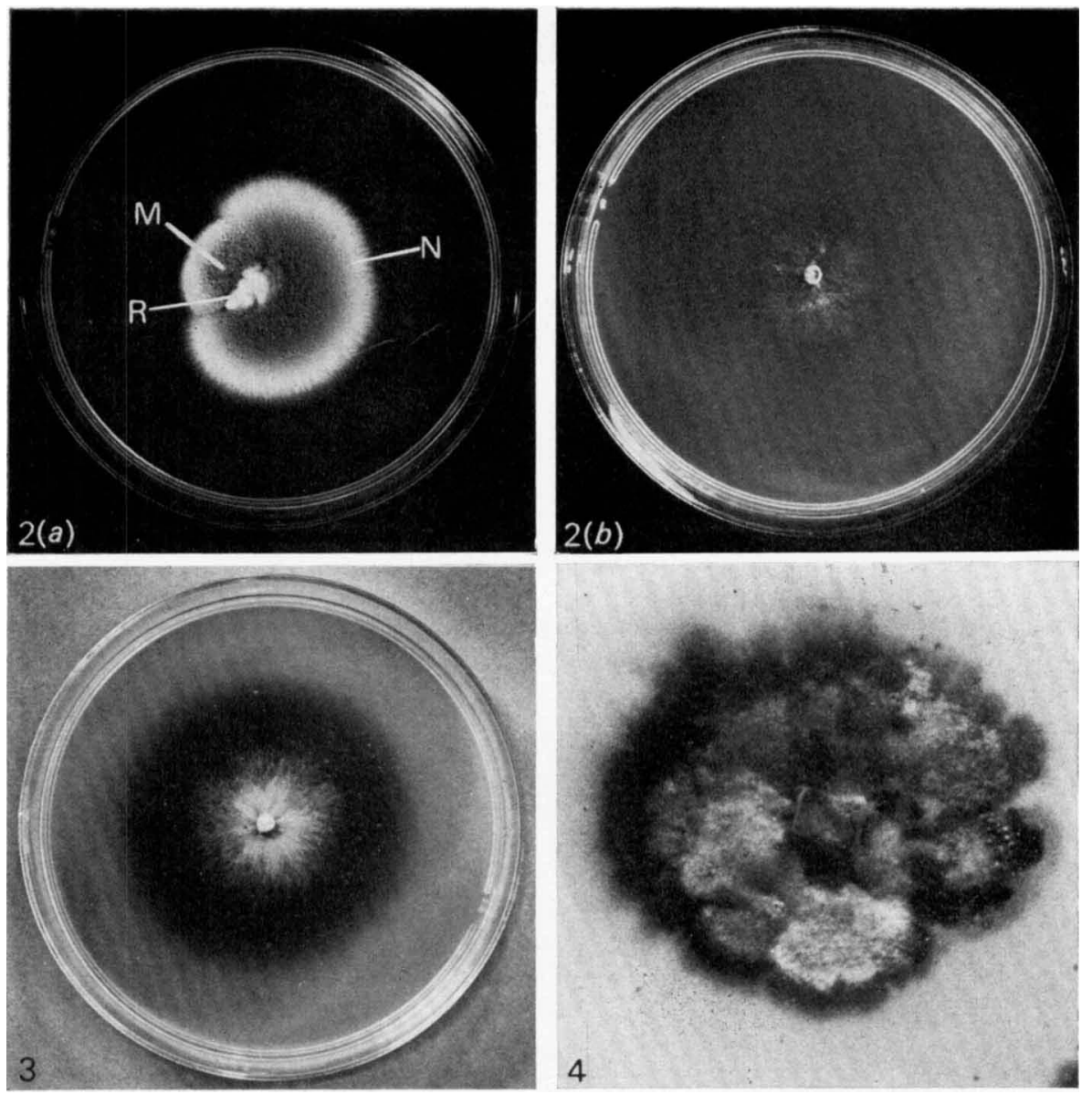

Fig. 2. Aspergillus nidulans strain N3 (pabaI, $y ;$ lysI) + A. rugulosus strain RI (proI) heterokaryon cultured on ( $a$ ) CM for 7 days; and $(b)$ MM for 10 days. The colony on CM $(a)$ shows segregation of $A$. nidulans $(\mathrm{N}), A$. rugulosus $(\mathrm{R})$ and mixed (M) sectors.

Fig. 3. A 'hybrid' colony from A. nidulans strain N1 (pabaI, y; pro2) $+A$. rugulosus strain $\mathrm{R} 2$ (metI) cultured on CM for 10 days.

Fig. 4. Aspergillus nidulans strain NI (pabaI, y; proz) +A. rugulosus strain R2 (metI) 'hybrid' on CM supplemented with benomyl $\left(\mathrm{I} \cdot 25 \mu \mathrm{g} \mathrm{ml}^{-1}\right)$ after Io days. The colony shows development of parental and recombinant sectors.

\section{Table 3. Colony development from protoplasts derived from heterokaryons and regenerated on $C M$}

Protoplasts were prepared from heterokaryons by the standard method. The NI +R2 heterokaryon was cultured for $24 \mathrm{~h}$ prior to protoplast preparation and the $\mathrm{N} 3+\mathrm{RI}$ heterokaryon for $72 \mathrm{~h}$. The inocula were derived from both 7- and 12-day-old cultures of heterokaryon NI +R2, and from a 7-day-old culture of heterokaryon $\mathrm{N}_{3}+\mathrm{RI}$.

Percentage of colonies of type:

$\begin{array}{ccccc}\text { Heterokaryon } & \begin{array}{c}\text { Inoculum age } \\ \text { (days) }\end{array} & \text { NI or N3 } & \text { RI or R2 } & \text { 'Hybrid' } \\ \text { NI + R2 } & 7 & 28 \cdot 6 & 60 \cdot 3 & \text { I1. I } \\ \text { NI + R2 } & 12 & 25 \cdot 5 & 47 \cdot 4 & 27 \cdot 1 \\ \text { N3 + R1 } & 7 & 31 \cdot 7 & 37 \cdot 7 & 30 \cdot 7\end{array}$


Table 4. Frequency (\%) of different colony types among single conidial descendants of the 'hybrids'

Conidial suspensions (about $1 \times 10^{5}$ conidia $\mathrm{ml}^{-1}$ ) were prepared from 10-day-old cultures grown on CM and MM. The values are means of results for at least four 'hybrid' colonies, with 10 platings for each colony. The frequency of recovery of parental or prototrophic recombinant colonies was inflated by the production of many more conidia in individual conidial heads than in those of the 'hybrid'.

\begin{tabular}{|c|c|c|c|}
\hline \multirow[b]{2}{*}{ Hybrid } & \multirow[b]{2}{*}{ Colony type } & \multicolumn{2}{|c|}{$\begin{array}{l}\text { Conidia derived from 'hybrid' } \\
\text { grown on: }\end{array}$} \\
\hline & & $\mathbf{M M}$ & $\mathbf{C M}$ \\
\hline $\mathrm{N} 3+\mathrm{RI}$ & $\begin{array}{l}\text { 'Hybrid' } \\
\text { N3 } \\
\text { RI } \\
\text { Other segregants }\end{array}$ & $\begin{array}{l}96 \cdot 01 \pm 3 \cdot 45 \\
0 \cdot 89 \pm 0 \cdot 67 \\
3 \cdot 11 \pm 2 \cdot 31 \\
0\end{array}$ & $\begin{array}{r}49 \cdot 91 \pm 5 \cdot 20 \\
10.55 \pm 4.91 \\
39 \cdot 54 \pm 9.84 \\
<0.1\end{array}$ \\
\hline$N I+R 2$ & $\begin{array}{l}\text { 'Hybrid' } \\
\text { NI } \\
\text { R2 } \\
\text { Green conidiated prototroph } \\
\text { Auxotrophic recombinants and other segregants }\end{array}$ & $\begin{array}{l}84 \cdot 02 \pm 8 \cdot 89 \\
0 \\
0 \\
15 \cdot 98 \pm 4 \cdot 31 \\
0\end{array}$ & $\begin{array}{r}60 \cdot 91 \pm 7 \cdot 24 \\
1 \cdot 97 \pm 0 \cdot 66 \\
3 \cdot 75 \pm 1 \cdot 76 \\
33 \cdot 36 \pm 5 \cdot 70 \\
<0 \cdot 1\end{array}$ \\
\hline $\mathrm{N} 3+\mathrm{R} 2$ & $\begin{array}{l}\text { 'Hybrid' } \\
\mathrm{N} 3 \\
\mathrm{R} 2 \\
\text { Green conidiated prototroph } \\
\text { Auxotrophic recombinants and other segregants }\end{array}$ & $\begin{array}{l}87 \cdot 81 \pm 7 \cdot 81 \\
0 \\
0 \\
12 \cdot 18 \pm 1 \cdot 72 \\
0\end{array}$ & $\begin{array}{r}36 \cdot 19 \pm 2 \cdot 55 \\
12 \cdot 85 \pm 4 \cdot 62 \\
2 \cdot 71 \pm 0 \cdot 74 \\
48 \cdot 09 \pm 6 \cdot 29 \\
<0.1\end{array}$ \\
\hline
\end{tabular}

Table 5. Number of nuclei, DNA content and DNA content per nucleus of parental and 'hybrid' protoplasts

\begin{tabular}{|c|c|c|c|c|}
\hline Strain & $\begin{array}{l}\text { No. of nuclei } \\
\text { per protoplast }\end{array}$ & $\begin{array}{c}\text { DNA content } \\
\text { per } 10^{6} \text { protoplasts } \\
(\mu \mathrm{g})\end{array}$ & $\begin{array}{c}\text { DNA content } \\
\text { per nucleus } \\
(\mu \mathrm{g})\end{array}$ & $\begin{array}{l}\text { Ratio DNA content } \\
\text { 'hybrid'/parent }\end{array}$ \\
\hline NI & $I \cdot 50 \pm 0 \cdot 18$ & 7.05 & $4.70 \times 10^{-6}$ & $2 \cdot 29$ \\
\hline & $2 \cdot 16 \pm 0.32$ & $1 \mathrm{I} \cdot 75$ & $5.43 \times 10^{-6}$ & $\mathrm{r} \cdot 98$ \\
\hline NI + R2 "hybrid" & $2 \cdot 79 \pm 0.27$ & $30 \cdot 10$ & $10^{\circ} 78 \times 10^{-6}$ & \\
\hline
\end{tabular}

Table 6. Complementation between segregants from ' hybrids' and parental or other auxotrophic strains

Mixtures of protoplasts from segregants and protoplasts from parental or other auxotrophic strains were treated with PEG and protoplasts from the fusion mixtures were plated on to MM.

\begin{tabular}{|c|c|c|c|c|}
\hline \multicolumn{2}{|c|}{ Segregant } & \multicolumn{2}{|c|}{ Tester strain } & \multirow[b]{2}{*}{ Complementation* } \\
\hline Code & Genotype & No. & Genotype & \\
\hline \multicolumn{5}{|c|}{ Segregants from N3+RI 'hybrid' } \\
\hline 1 & pabar;lysI & N3 & pabar, $y ;$ lysI & - \\
\hline $\mathbf{I}$ & pabar; lyst & RI & pror & + \\
\hline 2 & pror & N3 & pabar, $y ;$ lysI & + \\
\hline 2 & proI & $\mathbf{R I}$ & pror & - \\
\hline $\mathbf{I}$ & pabar; lyst & $\mathbf{R} \mathbf{I}$ & proI & + \\
\hline \multicolumn{5}{|c|}{ Segregants from NI + R2 'hybrid' } \\
\hline 3 & pabar, y;pro2, metr & N3 & pabar, $y ;$ lysi & - \\
\hline 3 & pabar, y;proz, metr & $\mathbf{R 2}$ & metI & - \\
\hline
\end{tabular}


A further group of segregants exhibited the nutritional requirements of the two parents, i.e. pabaI lysi or pror, but possessed colony morphologies and pigmentation different from the parents. These occurred at a frequency of $<0.1 \%$.

Conidia from the NI +R2 'hybrid' grown on MM produced 'hybrid' cultures and a new recombinant type which was prototrophic and green-spored. When grown on [CM the 'hybrid ' produced predominantly 'hybrid' subcultures and the same prototrophic recombinant. The parental types were also recovered although at frequencies lower than in the previous cross (Table 4). The prototrophic recombinant showed a mixture of characters of the two parents; spore colour and abundant cleistothecial production being typical of $A$. rugulosus while the spreading growth habit and intense conidiation were features of $A$. nidulans. Another recombinant type which was auxotrophic for all the markers, i.e. pabaI, $y$, pro2, metI, was also isolated, together with segregants which carried the nutritional requirements of the parents but varied colony morphologies.

Conidia from the $\mathrm{N} 3+\mathbf{R} 2$ 'hybrid' gave a similar pattern of segregation to those from the $\mathrm{NI}+\mathrm{R2}$ 'hybrid' (Table 4). For hybrids grown on CM the ratio of parental segregants was higher than in the previous crosses, with more $A$. nidulans types. Prototrophic and auxotrophic segregants, including a new lys $I$, met $I$ recombinant were also obtained.

Considering the various segregants isolated, all yellow-spored strains required $p$-aminobenzoic acid. This might be expected in view of the location of the $y$ and paba markers in linkage group I of $A$. nidulans. However, several segregants were isolated which carried the paba marker but were not yellow-spored. In these instances it is presumed that some interaction influenced pigment production.

The large size of conidia formed early in the growth of the 'hybrids' suggested that these cultures were diploid; however, the poor sporulation made it difficult to do confirmatory DNA estimations on conidia. Determinations of the DNA content and of the number of nuclei were made, however, on protoplasts prepared from the 'hybrids'. The data support the view that the 'hybrids' are diploid (Table 5). The different numbers of nuclei recorded might suggest a size differential between protoplasts from parental and 'hybrid' cultures, assuming the constant relationship between DNA content and cytoplasmic volume found with mycelial cells and conidia (Clutterbuck, 1969) to hold also in this instance. Protoplast volume was not determined in these studies. However, in a comparison of protoplasts from haploid and diploid strains of $A$. nidulans, protoplast size was not related to ploidy (S. Isaac, unpublished work). This suggests that the cytoplasm is fragmented in a random manner during protoplast formation. Furthermore the nuclear: cytoplasmic ratio of a particular protoplast may have implications for its future development.

Further evidence for diploidy was obtained from 'hybrids' cultured on CM supplemented with benomyl at $\mathrm{I} \cdot 0$ to $\mathrm{I} \cdot 5 \mu \mathrm{g} \mathrm{ml}^{-1}$ (Hastie, 1970) when segregation of parental and recombinant sectors was observed (Fig. 4). A great variety of segregants was isolated, including cultures auxotrophic for one or more markers of the parent strains and prototrophic cultures showing a wide range of morphological alteration from the parental types and similar to those obtained by spontaneous segregation. Confirmation that the auxotrophic segregants were carrying parental alleles was obtained by complementation tests involving back-fusion of the segregant with parental or other mutant strains (Table 6).

The evidence obtained indicates that the 'hybrids' are truly interspecific and arise from fusion of the different parental nuclei in the heterokaryon. This result suggests that genetic interaction is more likely in closely related species unlike the situation where more distally related species are used (Anné et al., 1976; Ferenczy et al., 1977).

F.K. acknowledges a fellowship from the British Council awarded on the basis of the British-Hungarian scientific exchange programme. J.F.P. is grateful to the S.R.C. for a research support grant (B/RG 4 I I 76). The authors thank Dr C. E. Caten for helpful advice in preparing this paper. 


\section{REFERENCES}

AnNé, J. \& Peberdy, J. F. (1975). Condition for induced fusion of fungal protoplasts in polyethylene glycol solutions. Archives of Microbiology 105, 201-205.

ANNE, J. \& PEBERdy, J. F. (1976). Induced fusion of fungal protoplasts following treatment with polyethylene glycol. Journal of General Microbiology 92, 413-417.

Anné, J., Eyssen, H. \& De SOMER, P. (1976). Somatic hybridization of Penicillium roquefortii with $P$. chrysogenum after protoplast fusion. Nature, London 262, 719-721.

Barron, G. L. \& MacNeIL, B. H. (1962). A simplified procedure for demonstrating the parasexual cycle in Aspergillus nidulans. Canadian Journal of Botany 40, 132 I-1327.

Bostock, G. J. (1970). DNA synthesis in the fission yeast Schizosaccharomyces pombe. Experimental Cell Research 60, 16-26.

Carlson, P.S. (1973). The use of protoplasts for genetic research. Proceedings of the National Academy of Sciences of the United States of America 70, 598-602.

Clutterbuck, A. J. (1969). Cell volume per nucleus in haploid and diploid strains of Aspergillus nidulans. Journal of General Microbiology 55, 291-299.

Clutterbuck, A. J. \& Roper, J. A. (1966). A direct determination of nuclear distribution in heterokaryons of Aspergillus nidulans. Genetical Research, Cambridge 7, $185-188$.

Cocking, E. C. (1972). Plant cell protoplasts isolation and development. Annual Review of Plant Physiology 23, 29-50.
DODGE, B. O. (1936). Interspecific hybrids involving factors for ascus abortion in Neurospora. American Journal of Botany 23, 555-56I.

Ferenczy, L., Kevei, F. \& Szegedi, M. (1975a). High-frequency fusion of fungal protoplasts. Experientia 31, 1028-1029.

Ferenczy, L., KeVeI, F. \& Szegedi, M. (1975b). Increased fusion frequency of Aspergillus nidulans protoplasts. Experientia 31, 50-52.

FERENCZy, L., SZegedi, M. \& KeVei, F. (1977). Interspecific protoplast fusion and complementation in Aspergilli. Experientia 33, 184-186.

Hastie, A. C. (1970). Benlate induced instability of Aspergillus diploids. Nature, London 226, 771 .

Hastie, A. C. (1973). Hybridization of Verticillium albo-atrum and Verticillium dahliae. Transactions of the British Mycological Society 60, 51 I-523.

LEWIS, L. A. (1969). Genetic evidence for hybridization in an interspecific cross in the genus Sordaria. Journal of General Microbiology 59, 359-367.

Peberdy, J. F. \& IsaAC, S. (1976). An improved procedure for the isolation of protoplasts from Aspergillus nidulans. Microbios Letters 3, 7-9.

Pontecorvo, G., Roper, J. A., Hemmons, D. W., MacDonald, K. D. \& Bufton, A. W. (1953). The genetics of Aspergillus nidulans. Advances in Genetics 5, 141-238.

RAPER, K. B., \& Fennell, D. (1965). The genus Aspergillus. Baltimore: Williams \& Wilkins.

Ruddle, F. H. \& Creagan, R. P. (1975). Parasexual approaches to the genetics of man. Annual Review of Genetics 9, 407-486.

Wessels, J. G. H. (1965). Morphogenesis and biochemical processes in Schizophyllum commune Fr. Wentia 13, 1-1 13. 